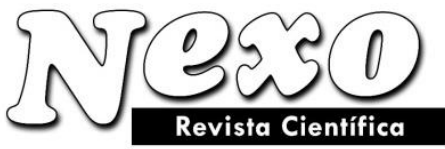

ISSN-E 1995-9516

Universidad Nacional de Ingeniería http://revistas.uni.edu.ni/index.php/Nexo http://dx.doi.org/10.5377/nexo.v29i01.4396

\title{
ESTUDIO DE UN PROTOCOLO PARA DETERMINAR AUTOXIDACION EN LIPIDOS DE PRODUCTOS DE PESCADO DE RIO
}

\section{STUDY OF A PROTOCOL FOR DETERMINING AUTOXIDATION IN LIPIDS OF FRESHWATER FISH PRODUCTS}

\author{
J.R. Medina ${ }^{1, *}$, A. Melica ${ }^{1}$, J. Lorenzati ${ }^{1}$, G.A. Pérez ${ }^{1}$ \\ ${ }^{1}$ Universidad Nacional del Litoral. Facultad de Ingeniería Química - UNL - Santiago del Estero 2829 \\ S3000 AOM Santa Fe- Argentina. \\ *jrmedina@ fiq.unl.edu.ar
}

\begin{abstract}
RESUMEN
En Argentina, la especie de río de agua dulce más abundante pero sin valor comercial es el sábalo (Prochilodus platensis), por este motivo se ha desarrollado tecnologías de aprovechamiento de dicha especie. Sin embargo, su valor lipídico más alto en comparación a los peces de mar, acelera el proceso de oxidación, afectando sus propiedades y su calidad sensorial. Aquí se implementará un esquema metodológico adecuado para evaluar la autoxidación en el músculo fresco de sábalo sin procesar y su concentrado proteico, ambos conservados a $-20^{\circ} \mathrm{C}$, siguiendo el deterioro según el índice de peróxidos, compuestos reactivos al ácido tiobarbitúrico e hierro hemínico. Se encontró que la determinación de TBARS es más eficiente el desarrollo de color a temperaturas de ebullición, y un incremento en la concentración del reactivo TBA no mejora significativamente la señal de absorbancia. La presencia de crioprotectores es una limitante a la técnica TBARS, el cual provoca importantes interferencias. El nivel de hierro hemínico presenta una disminución proporcional según sea el tiempo de incubación y la cocción de las muestras. Los niveles de peróxidos en muestras de músculo fresco y surimi son muy bajos, implicando que si la conservación es controlada se evita este deterioro
\end{abstract}

Palabras claves: Surimi, Filet, Autoxidación, Peróxidos, TBA.

\begin{abstract}
In Argentina, the species of freshwater river more abundant but without commercial value is the sábalo (Prochilodus platensis), for that reason have been developed technologies of use of this species. However, its higher lipid value compared to sea fish accelerates the oxidation process, affecting its properties and sensory quality. Here, a suitable methodological scheme will be implemented to evaluate the autoxidation in the fresh muscle of unprocessed sardine and its protein concentrate, both conserved at $-20^{\circ} \mathrm{C}$, following the deterioration according to the peroxide index, compounds reactive to thiobarbituric acid and heminic iron. It was found that the determination of TBARS is more efficient the color development at boiling temperatures, and an increase in the concentration of the TBA reagent does not significantly improve the absorbance signal. The presence of cryoprotectants is a limitation to the TBARS technique, which causes significant interference. The heminic iron level presents a proportional decrease according to the incubation time and the cooking of the samples. The levels of peroxides in samples of fresh muscle and surimi are very low, implying that if conservation is controlled this deterioration is avoided
\end{abstract}

Keywords: FPC, Heme iron, Sábalo, Peroxide value, TBAR 


\section{INTRODUCCIÓN}

El concentrado proteico de pescado (CPP) o "surimi" no es un alimento en sí mismo, sino que es el intermediario para formulaciones de sustitutos de mariscos. Los principales motivos del auge del CPP a nivel mundial son: el mejor aprovechamiento del recurso ictícola desde el punto de vista nutricional, la versatilidad tecnológica ya que involucra una tecnología simple y de relativamente bajos costos de inversión, ofreciendo a la vez la posibilidad de estabilizar la textura de la pulpa mediante el agregado de crioprotectores, y por último la expansión de mercados existentes y la alternativa de desarrollar nuevos productos atractivos que permitirá la ampliación del mercado de productos pesqueros (Manca y Trinchero, 1984a).

Medina (2000), Medina y Garrote (2002) y Medina et al. (2008) en su línea de investigación han planteado con sostenido interés la posibilidad de utilizar como materia prima en la obtención de CPP, especies provenientes de agua dulce, las cuales presentan disponibilidad de captura, como es el surubí, y actualmente el sábalo.El sábalo (Prochilodus platensis) es el recurso pesquero más abundante del litoral fluvial argentino. Es un eslabón crucial en los ecosistemas que integra, dada su condición de especie forrajera, sostén de la cadena trófica. Se trata de un pez iliófago.

Es una especie naturalmente muy fecunda, y la misma forma parte de una estrategia reproductiva exitosa, adaptada a las características del régimen natural de pulsos de inundación del sistema, que involucra la realización de migraciones río arriba y el desove en aguas abiertas, acoplado a las crecientes, como mecanismo de dispersión de huevos y larvas a las áreas de cría del valle aluvial (Espinach Ros y Sánchez, 2007)

En nuestra región, una de las especies de río más abundantes pero que no posee un elevado valor en el mercado es el sábalo, por este motivo se ha desarrollado la tecnología de surimi en dicha especie. Sin embargo se presenta como inconveniente, su valor lipídico más alto en comparación a los peces de mar que se usan para surimi, por lo que se acelera el proceso de oxidación, afectando sus propiedades y calidad sensorial.

La oxidación de los lípidos es la segunda causa de deterioro de los alimentos, después de la acción de los microorganismos; por lo que representa de gran interés económico para la industria alimenticia ya que tiene como consecuencias las alteraciones en el aroma y sabor (enranciamiento), en el color, la pérdida de determinados nutrientes y la formación de substancias potencialmente nocivas. La forma principal de oxidación de los lípidos es mediante una reacción de propagación en cadena de radicales libres, en la que a partir de ácidos grasos y oxígeno se van formando hidroperóxidos mientras quede oxígeno y ácidos grasos oxidables. Estos hidroperóxidos son relativamente inestables e intervienen en reacciones que dan lugar a la formación de ciertos aldehídos, cetonas, alcoholes, entre otros.

Debido a la naturaleza no homogénea de la matriz con la que se trabajará es que no hay buenos métodos para evaluar la oxidación de los lípidos.

En el presente trabajo se plantea implementar un esquema metodológico que resulte adecuado para investigar la autoxidación en el músculo de sábalo sin procesar y el concentrado proteico de sábalo (surimi), ambos conservados a temperatura de congelación $\left(-20^{\circ} \mathrm{C}\right)$. Se realizará el seguimiento de ciertos parámetros que caracterizan el deterioro en el estado congelado; algunos de ellos son: índice de peróxidos, compuestos reactivos al ácido tiobarbitúrico (TBAs) e hierro hemínico. 


\section{MATERIALES Y METODOS}

\subsection{Obtención y preparación de las muestras de sábalo fresco}

Los ejemplares de sábalo obtenidos pesaban 2- $3 \mathrm{~kg}$, y medían 40-60 cm de longitud, capturados en las cercanías de la ciudad de Santa Fe, Argentina.

Luego de ser lavado con agua corriente, se eliminaron espinas, escamas, vísceras y sangre. Se cortaban filetes, seleccionando el músculo blanco y posteriormente se procedió a picar con picadora eléctrica de cuchillas. Se envasó el material picado en vasos de polipropileno con tapa y filetes íntegros se almacenaron en bolsas de polietileno de alta densidad. La temperatura de almacenamiento fue de $-21{ }^{\circ} \mathrm{C}$ en freezer. Se trabajó con tres tipos de muestras, músculo de sábalo sin procesar y dos fracciones de concentrado de proteínas de sábalo (surimi) elaboradas mediante la técnica de Medina et al. (2010).

\subsection{Métodos analíticos:}

Determinación del Índice de Peróxidos (PV): Este es un método colorimétrico indirecto. Se basa en que a una muestra que contenga peróxidos se adiciona un reactivo de hierro (II); en la muestra se llevará a cabo la oxidación electroquímica de hierro (II) a hierro (III) (Jiang et al, 1992) y éste último será cuantificado por su reacción de complejación con tiocianato mostrando un color rojo característico. (Kirk, 1991).

$$
\begin{gathered}
\mathrm{Fe}^{2+}+\mathrm{ROOH} \rightarrow+\mathrm{Fe}^{3+} \\
\mathrm{Fe}^{3+}+\mathrm{SCN}^{-} \rightarrow(\mathrm{FeSCN})^{2+}
\end{gathered}
$$

Una muestra de 2 gr es colocada en una solución de cloroformo/metanol (2:1), llevándola a un volumen de $10 \mathrm{ml}$, posteriormente se agita y filtra. Se adiciona $0,05 \mathrm{ml}$ de una solución de tiocianato de amonio (30\% $\mathrm{m} / \mathrm{v}$ ), se mezcla y mide absorbancia a $500 \mathrm{~nm}$ frente a un blanco de solventes. (E0). Luego se agrega 0,05 $\mathrm{ml}$ de solución de cloruro ferroso al $0,35 \%$ con $2 \%$ de $\mathrm{HCl} 10 \mathrm{M}$, que dará un complejo coloreado, leyendo nuevamente a $500 \mathrm{~nm}$ pasados unos 5 minutos (E2). Simultáneamente hacer una determinación del blanco (E1).

Se realizó una curva de calibrado con cloruro férrico en concentraciones que contenían entre 5 y $50 \mu \mathrm{g}$ de $\mathrm{Fe} / 10 \mathrm{ml}$. Estas se prepararon mezclando $10 \mathrm{ml}$ de cada dilución de cloruro férrico con $0,05 \mathrm{ml}$ de tiocianato de amonio y $0,05 \mathrm{ml}$ de $\mathrm{HCl} \mathrm{0,2M}$, leyendo a la misma longitud de onda (500 $\mathrm{nm}$ ).

El índice de peróxidos (meq/kg) se obtiene relacionando la cantidad de Fe, expresados como $\mu \mathrm{g} / 10 \mathrm{ml}$ obtenidos en la reacción, con el peso de la muestra. En la Figura 1 se puede ver la curva de calibrado que relaciona las Absorbancias determinadas para las distintas concentraciones de Fe una vez corregidas por los blancos $(\mathrm{E} 2-(\mathrm{E} 1+\mathrm{E} 0))$

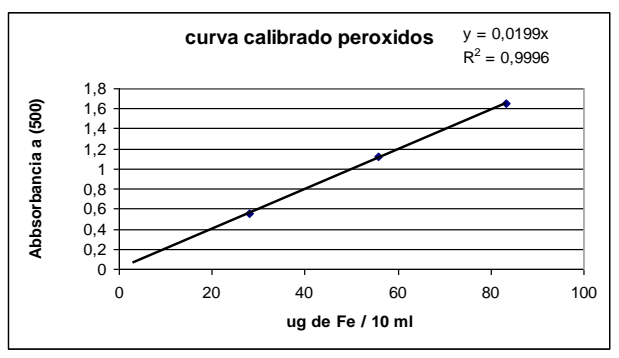

Figura 1: curva de calibrado para el índice de peróxidos. 


\section{J.R. Medina et al.}

Sustancias reactivas al ácido tiobarbitúrico (sigla en inglés: TBARS: siguiendo la técnica de Siu and Draper (1978), se realizaron varias experiencias con el objeto de encontrar las condiciones óptimas de trabajo. Se realizaron pruebas a distintas temperaturas, tiempos de reacción y concentraciones de TBA. Se trabajó con tres tipos de muestras, músculo de sábalo sin procesar y dos fracciones de surimi elaboradas, mediante la técnica de Medina et al. (2010), bajo las mismas condiciones a partir de un mismo sábalo pero conservadas en envolturas de papel aluminio diferentes. Otro de los ensayos realizados consistió en observar el desarrollo oxidativo que se produce en músculo de sábalo fresco y cocido cuando las muestras son sometidas a un tratamiento térmico suave $\left(37^{\circ} \mathrm{C}\right)$ durante distintos períodos de tiempo.

Previamente, se empleó la técnica de Wang et al.(2002) con el mismo objetivo la cual tuvo que descartarse debido a que en primera instancia no se pudieron analizar las muestras ya que se observaba una elevada turbidez que imposibilitaba usar el espectrofotómetro y, por otro lado uno de los reactivos usados (terbutilhidroquinona TBQH) inhibía la oxidación de la muestra imposibilitando la generación del color característico.

Un fragmento de 20 gramos de muestra fue molido con procesadora y homogeneizado con $50 \mathrm{ml}$ de agua destilada. Luego se adicionan $50 \mathrm{ml}$ de TCA al $10 \%$ mezclándose en agitador y posteriormente se filtra reservando el sobrenadante. Se tomó una alícuota de $4 \mathrm{ml}$ del filtrado y se le adicionó $1 \mathrm{ml}$ de ácido tiobarbitúrico, de concentración variable $20 \mathrm{mM}, 40 \mathrm{mM}$ y $80 \mathrm{mM}$, por sucesivas diluciones estabilizando la solución $80 \mathrm{mM}$ llevándola a pH 4 con ácido clorhídrico; en tubos con tapa a rosca. Se incubaron los tubos en baños de agua a $35^{\circ} \mathrm{C}$ por 24 horas, $60^{\circ} \mathrm{C}$ por 90 minutos y a $100^{\circ} \mathrm{C}$ por 30 minutos. Se enfriaron los tubos con agua corriente y se leyeron la absorbancia a $532 \mathrm{~nm}$.

La curva de calibrado fue construida incubando diferentes concentraciones de MDA, preparado según Wang et al.(2002) en una solución que contiene $2 \mathrm{ml}$ de agua destilada, $2 \mathrm{ml}$ de TCA $10 \%$ y $1 \mathrm{ml}$ de TBA 20mM. Esta se lleva a baño maría a temperatura de ebullición durante 15 minutos. Se traza la curva midiendo absorbancia a $532 \mathrm{~nm}$.

Hierro hemínico: es determinado mediante el método de Hornsey (1956). Una muestra de 2 gr es colocada en un tubo Falcon y se le adiciona $9 \mathrm{ml}$ de acetona acida ( $90 \%$ acetona, $8 \%$ de agua destilada y $2 \% \mathrm{HCl}$ ). Se deja macera y deja en reposo durante una hora en lugar oscuro y a temperatura ambiente. El extracto es filtrado y se lee la absorbancia a $640 \mathrm{~nm}$ contra un reactivo de acetona acida como blanco. Los pigmentos totales, como la hematina acida son calculados con la fórmula:

Pigmentos totales $(\mathrm{ppm})=\mathrm{A} 640 * 680$

Y el hierro hemínico es calculado como:

Hierro hemínico $(\mathrm{ppm})=$ pigmentos totales $* 8,82 / 100$

\section{RESULTADOS}

En general, luego del tiempo de incubación para todas las experiencias se desarrolla un complejo de color rosado el cual es más intenso a mayores temperaturas, como se demuestra en la tabla 1. El tiempo para la incubación de $35^{\circ} \mathrm{C}$ fue de 24 hs mientras que para la temperatura de ebullición fue de 30 minutos.

Además, se observa que un aumento en la concentración del reactivo TBA no genera una variación significativa en la concentración de MDA, por lo que no se justifica el gasto excesivo de éste. 


\section{J.R. Medina et al.}

Tabla 1: Absorbancia correspondiente a TBARS en músculo fresco de sábalo a distintas temperaturas y concentraciones de TBA.

\begin{tabular}{ccc}
\hline \multirow{2}{*}{ Ácido tiobarbitúrico } & \multicolumn{2}{c}{ Absorbancia } \\
& $T=35^{\circ} \mathrm{C}$ & $T=100^{\circ} \mathrm{C}$ \\
\hline $20 \mathrm{mM}$ & 0,090 & 0,194 \\
$40 \mathrm{mM}$ & 0,108 & 0,214 \\
$80 \mathrm{mM}$ & 0,114 & 0,225 \\
\hline
\end{tabular}

En la tabla 2 se observa el mismo comportamiento que para el músculo tratado anteriormente, el aumento en la concentración del TBA no genera grandes variaciones en la concentración de MDA.

En un principio, las fracciones fueron tratadas para obtener datos por duplicado, sin embargo hubo que tomarlas individualmente ya que los niveles de oxidación discreparon bastante; esto pudo ser porque la incorporación de los crioprotectores fue hecha en forma manual, no garantizándose la homogeneidad en cada una de las fracciones.

Dado que las muestras poseen un porcentaje de crioprotectores (sacarosa - sorbitol 1:1 al 8\% del peso total) cuando la temperatura de los baños fue de 60 y $100^{\circ} \mathrm{C}$ se formó un complejo amarillo-rosado con picos de absorbancia máxima a 450 y $532 \mathrm{~nm}$, respectivamente. Esto ocurrió debido a la presencia de sacarosa, quien generó reacciones de interferencia. A bajas temperaturas no hubo presencia de este complejo, por lo que la interferencia se puede reducir trabajando a menores temperaturas a costa de aumentar el tiempo de la reacción La intensidad del color amarillo fue mayor a $100^{\circ} \mathrm{C}$, produciendo valores de absorbancia mayores a $450 \mathrm{~nm}$ que a $532 \mathrm{~nm}$, demostrando que la interferencia por sacarosa era superior a los niveles de TBARs. Para evitar esto último, una opción sería determinar la concentración de MDA por destilación del mismo.

Tabla 2: Absorbancia correspondiente a TBARs en dos muestras de surimi de Sábalo a distintas temperaturas y concentraciones de TBA.

\begin{tabular}{|c|c|c|c|c|c|c|c|c|c|c|c|c|}
\hline \multirow{5}{*}{$\begin{array}{c}\text { Acido } \\
\text { tiobarbitúrico }\end{array}$} & \multicolumn{6}{|c|}{ Muestra 1} & \multicolumn{6}{|c|}{ Muestra 2} \\
\hline & \multicolumn{6}{|c|}{ Absorbancia } & \multicolumn{6}{|c|}{ Absorbancia } \\
\hline & \multicolumn{2}{|c|}{$T=35^{\circ} \mathrm{C}$} & \multicolumn{2}{|c|}{$T=60^{\circ} \mathrm{C}$} & \multicolumn{2}{|c|}{$T=100^{\circ} \mathrm{C}$} & \multicolumn{2}{|c|}{$T=35^{\circ} \mathrm{C}$} & \multicolumn{2}{|c|}{$T=60^{\circ} \mathrm{C}$} & \multicolumn{2}{|c|}{$T=100^{\circ} \mathrm{C}$} \\
\hline & 532 & 450 & 532 & 450 & 532 & 450 & 532 & 450 & 532 & 450 & 532 & 450 \\
\hline & $\mathrm{nm}$ & $n m$ & $n m$ & $\mathrm{~nm}$ & $n m$ & $n m$ & $n m$ & $n m$ & $n m$ & $n m$ & $n m$ & $n m$ \\
\hline $20 \mathrm{mM}$ & 0,189 & 0,053 & 0,218 & 0,194 & 0,261 & 0,537 & 0,153 & 0,031 & 0,189 & 0,156 & 0,187 & 0,385 \\
\hline $40 \mathrm{mM}$ & 0,196 & 0,078 & 0,228 & 0,214 & 0,275 & 0,522 & 0,176 & 0,081 & 0,195 & 0,16 & 0,266 & 0,447 \\
\hline $80 \mathrm{mM}$ & 0,215 & 0,09 & 0,259 & 0,225 & 0,268 & 0,513 & 0,167 & 0,06 & 0,164 & 0,147 & 0,261 & 0,45 \\
\hline
\end{tabular}

El tratamiento suave de las muestras en baño maría a $37^{\circ} \mathrm{C}$ generó un leve aumento en la oxidación cuando varía el tiempo de la incubación; comparando con las muestras cocidas este incremento es más acentuado, como se aprecia en la figura 2, debido a que han pasado por un tratamiento térmico más severo previamente (cocción a $90^{\circ} \mathrm{C}$ ). 


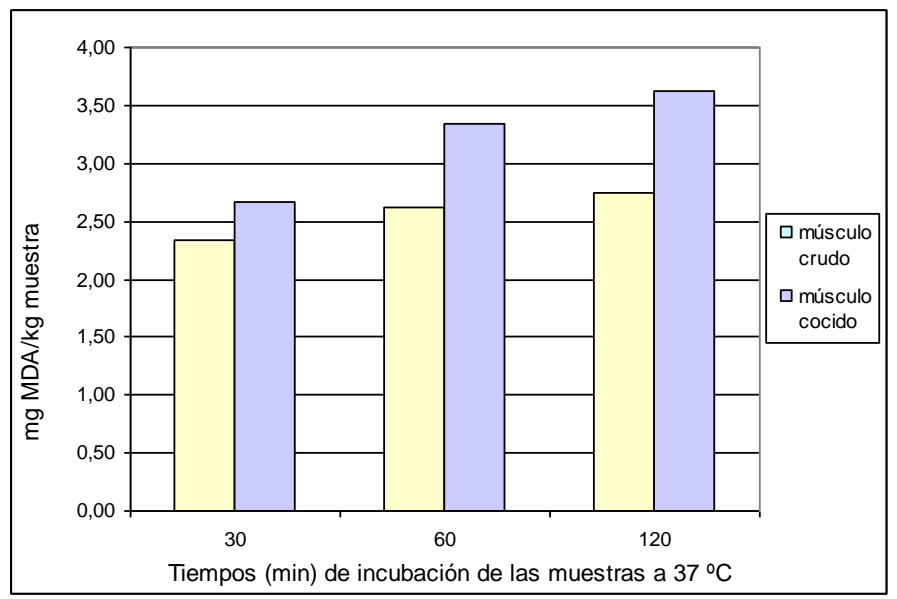

Figura 2: Concentraciones de malondialdehido para distintos tiempos de incubación a $37^{\circ} \mathrm{C}$ en músculo de sábalo crudo y cocido.

El hierro hemínico abunda en toda carne de origen animal y no solo es responsable del color sino que también está íntimamente relacionado con la nutrición y dieta diaria. Se ha demostrado que varios procesos, como el almacenamiento y cocción, incrementan la degradación del hierro hemínico oxidándolo a metamioglobina, generando defectos de color.

En la figura 3 se observa como el contenido de hierro hemínico disminuye con el tiempo de exposición de las muestras a $37^{\circ} \mathrm{C}$ y, más aún con el efecto de cocción. Esta disminución está asociada a la desnaturalización de la mioglobina, la cual aumenta con la temperatura.

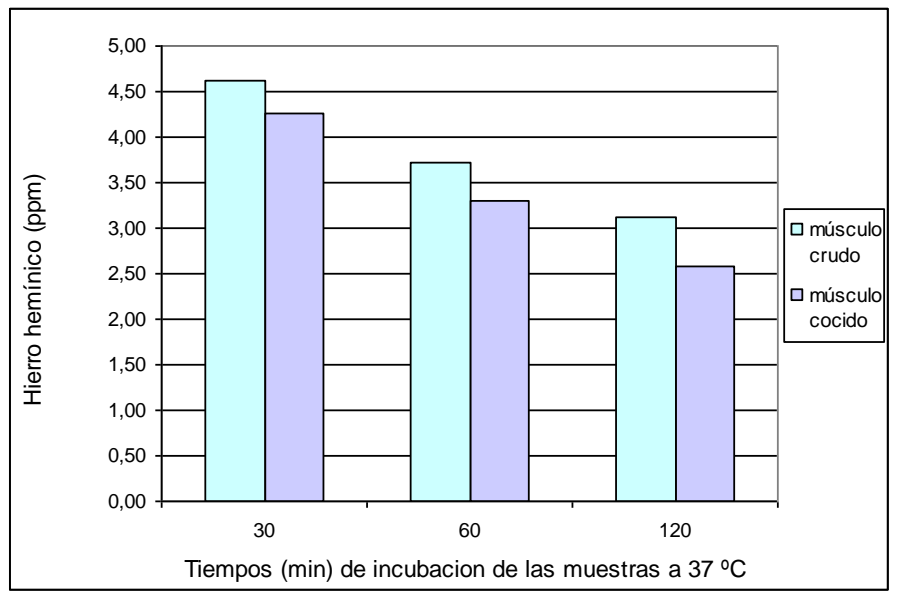

Figura 3: concentraciones de hierro hemínico en músculo de sábalo crudo y cocido para distintos tiempos de incubación a $37^{\circ} \mathrm{C}$.

Los valores de índice de peróxidos se encuentran expuestos en la tabla 5, y representan un promedio de duplicados, se observan bajos niveles de oxidación en ambas muestras. 


\section{J.R. Medina et al.}

Tabla 5: índice de peróxidos en ( $\mu \mathrm{g}$ Fe/ g muestra) para muestras de surimi y músculo de sábalo sin procesar.

\begin{tabular}{ccc}
\hline Muestra & $\begin{array}{c}\text { Absorbancia } \\
(\mathrm{nm})\end{array}$ & $\begin{array}{c}\text { Concentración } \\
(\mu \mathrm{g} \mathrm{Fe} / \mathrm{g} \\
\text { muestra })\end{array}$ \\
\hline músculo & 0,027 & 1,357 \\
surimi & 0,039 & 1,960 \\
\hline
\end{tabular}

\section{CONCLUSIONES}

Se encontró que en la determinación de TBARS es más eficiente el desarrollo de color a temperaturas de ebullición, con la ventaja de la disminución de los tiempos de reacción. Un incremento en la concentración del reactivo TBA no mejora significativamente la señal de absorbancia.

Se debe tener en cuenta como limitación a la técnica la presencia de elevadas concentraciones de sacarosa, como es en el caso del concentrado proteico de sábalo donde se incorpora como crioprotector, el cual provoca importantes interferencias.

El nivel de hierro hemínico se encuentra altamente influenciado por el tiempo de incubación de las muestras y tratamientos térmicos como es la cocción, donde se observa una disminución proporcional a tales tratamientos.

Los niveles de peróxidos en muestras de músculo fresco y surimi son muy bajos, lo que demuestra que si las condiciones de conservación son controladas es factible evitar este deterioro.

Por otro lado, las técnicas de determinación de peróxidos editadas en normas internacionales se aplican sobre materia grasa aislada, mientras que aquí se trabajó sobre la muestra desmenuzada, lo cual es una ventaja porque evita la inducción de la oxidación por la propia preparación de la muestra, descartando tratamientos agresivos.

\section{REFERENCIAS}

Espinach Ros, A. and Sánchez, R.P. (2007) (Eds.), Proyecto Evaluación del Recurso Sábalo en el Paraná Informe final de los resultados de la primera etapa 2005-2006 y medidas de manejo recomendadas, Secretaría de Agricultura, Ganadería, Pesca y Alimentos, Subsecretaría de Pesca y Acuicultura.

Fernández-López, J., L. Sevilla, E. Sayas-Barberá, C. Navarro, F. Marín, J.A. Pérez-Alvarez (2006). Evaluation of the Antioxidant Potential of Hyssop (Hyssopus officinalis L.) and Rosemary (Rosmarinus officinalis L.) Extracts in Cooked Pork Meat.

Jiang, Zhen-Yue; Hunt, J.V. y Wolff, SP. (1992) Ferrous ion oxidation in presence of xylenol orange for detection of lipid hydroperoxide in low density lipoprotein. Analytical Biochemistry, 202, 384-389

Kirk R. S., Sawyer R; Egan, H. Composición y análisis de alimentos de Pearson, segunda edición; Compañía editorial continental SA de CV, México, 1996.

Manca, E.A. y Trinchero, J.L. (1984). Desmenuzado de pescado: tecnología y posibles usos (Parte I). La Industria Cárnica Latinoamericana, Vol. 56, pp. 41-50. 


\section{J.R. Medina et al.}

Medina, J. R. and Garrote, R. L. (2002) Determining washing conditions during the preparation of frozen surimi from Surubí (Pseudoplatystome coruscans) using response surface methodology, Journal of Food Science, Vol. 67, No. 3, pp. 1455-1461.

Medina, J. R.; Reinheimer, M. A.; Freyre, M. R.; Pérez, G. A. (2010). Cambios en la composición y de los ácidos grasos del surimi elaborado a partir de sábalo (prochilodus platensis) modificando las condiciones de la etapa del lavado. Revista Mexicana de Ingeniería Química, vol. 9, núm. 2, pp. 159-166

Medina, J.R. (2000). Estudio de la etapa de lavado en la obtención de surimi congelado a partir de pescado de río. Tesis doctoral, MS thesis. Universidad Nacional del Litoral. Santa Fe, Argentina.

Medina, J.R.; Freyre, M.R.; Piagentini, M.A. y Pérez, G.A. (2008). Efecto de las condiciones del lavado sobre la composición de nutrientes en surimi obtenido a partir de pescado de río. En: XVII Congreso Brasilero de Ingeniería Química (XVII COBEQ).

Sadettin Turhan, N. Sule Ustun, Inci Bank. (2006). Effect of freeze-thawcycles on total and heme iron contents of bonito (Sarda sarda) and bluefish (Pomatomus saltator) fillets. J. Food Composition and Analysis. June, Pages 384-387

Siu, GM, and Draper, HH (1978). A survey of the malonaldehyde content of retail meats and fish. J. Food Sci. 43:1147-1149.

Wang, B. RD Pace, AP Dessai, A. Bovell-Benjamin and B. Phillips (2002). Modified extraction method for determining 2-thiobarbituric acid values in meat with increased specificity and simplicity. Journal of Food Science, 67, pp. 2833-2836.

\section{SEMBLANZA DE LOS AUTORES}

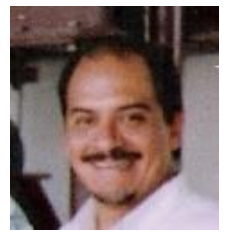

José R. Medina es Ingeniero Químico y Magister en Ciencia y Tecnología de Alimentos, recibido en la Universidad Nacional de Litoral (Argentina). Ha desarrollado varios proyectos de investigación en el área de tecnología de alimentos e ingeniería de procesos. Se desempeña como Profesor Adjunto responsable de la asignatura Proyecto Industrial y colabora en la asignatura de Ingeniería Económica de la Facultad de Ingeniería Química de la Universidad Nacional del Litoral, Santa Fe, Argentina, y actualmente trabaja en líneas de investigación vinculadas con la Tecnologías de procesamiento y conservación de matrices cárnicas.

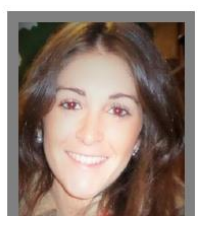

Josefina Lorenzatti, pasante de investigación y alumna de la carrera de grado Ingeniería en alimentos de la Facultad de Ingeniería Química de la Universidad Nacional del Litoral, Santa Fe, Argentina.

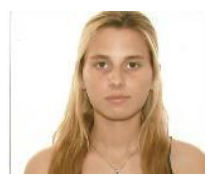

Agustina Melica, pasante de investigación y alumna de la carrera de grado Ingeniería en alimentos de la Facultad de Ingeniería Química de la Universidad Nacional del Litoral, Santa Fe, Argentina.

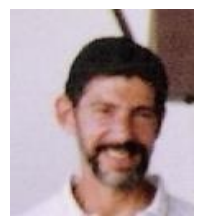

Gustavo A. Pérez es Ingeniero Químico, recibido en la Universidad Nacional del Litoral (Argentina). Desarrolla sus actividades como Profesor Titular en las asignaturas Ingeniería Económica y Proyecto Industrial en la Facultad de Ingeniería Química de la Universidad Nacional del Litoral. Además realiza tareas de investigación como experto en el área de "Ingeniería, simulación y optimización de Procesos de la industria química". 